\title{
A Newly Identified Prophage Gene, ymfM, Causes SOS-Inducible Filamentation in Escherichia coli
}

\author{
Shirin Ansari, a,b* James C. Walsh, ${ }^{\mathrm{b}}$ Amy L. Bottomley, a (D) lain G. Duggin, a (D) Catherine Burke, ${ }^{a}$ (D) Elizabeth J. Harry ${ }^{a}$ \\ aThe ithree institute, Faculty of Science, University of Technology Sydney, Sydney, Australia \\ bEMBL Australia Node in Single Molecule Science and ARC Centre of Excellence in Advanced Molecular Imaging, School of Medical Sciences, University of New South \\ Wales, Sydney, Australia
}

ABSTRACT Rod-shaped bacteria such as Escherichia coli can regulate cell division in response to stress, leading to filamentation, a process where cell growth and DNA replication continue in the absence of division, resulting in elongated cells. The classic example of stress is DNA damage, which results in the activation of the SOS response. While the inhibition of cell division during SOS has traditionally been attributed to SulA in E. coli, a previous report suggests that the e14 prophage may also encode an SOS-inducible cell division inhibitor, previously named SfiC. However, the exact gene responsible for this division inhibition has remained unknown for over 35 years. A recent high-throughput overexpression screen in $E$. coli identified the e14 prophage gene, ymfM, as a potential cell division inhibitor. In this study, we show that the inducible expression of $y m f M$ from a plasmid causes filamentation. We show that this expression of $y m f M$ results in the inhibition of $Z$ ring formation and is independent of the well-characterized inhibitors of FtsZ ring assembly in $E$. coli, SulA, SImA, and MinC. We confirm that ymfM is the gene responsible for the SfiC phenotype, as it contributes to the filamentation observed during the SOS response. This function is independent of SulA, highlighting that multiple alternative division inhibition pathways exist during the SOS response. Our data also highlight that our current understanding of cell division regulation during the SOS response is incomplete and raises many questions regarding how many inhibitors there actually are and their purpose for the survival of the organism.

IMPORTANCE Filamentation is an important biological mechanism that aids in the survival, pathogenesis, and antibiotic resistance of bacteria within different environments, including pathogenic bacteria such as uropathogenic Escherichia coli. Here, we have identified a bacteriophage-encoded cell division inhibitor which contributes to the filamentation that occurs during the SOS response. Our work highlights that there are multiple pathways that inhibit cell division during stress. Identifying and characterizing these pathways are critical steps in understanding survival tactics of bacteria, which become important when combating the development of bacterial resistance to antibiotics and their pathogenicity.

KEYWORDS Escherichia coli, SOS, SfiC, YmfM, cell division, e14 prophage, filamentation

acterial cell division is an essential process that is tightly regulated to ensure division occurs at the correct time and position in order to create two viable and genetically identical daughter cells (1). In Escherichia coli, this begins with the accumulation of the essential protein, FtsZ, into a ring-like structure ( $Z$ ring), at midcell (2). Following this, several downstream division proteins are recruited to form a complex, known as the divisome, which then constricts to divide the cell in two (3). There are several regulatory mechanisms that underlie the timing and positioning of division in E. coli. This includes the well-characterized Min system, which prevents the formation
Citation Ansari S, Walsh JC, Bottomley AL, Duggin IG, Burke C, Harry EJ. 2021. A newly identified prophage gene, ymfM, causes SOS Inducible filamentation in Escherichia coli. J Bacteriol 203:e00646-20. https://doi.org/10 .1128/JB.00646-20.

Editor Yves V. Brun, Université de Montréal Copyright $\odot 2021$ American Society for Microbiology. All Rights Reserved.

Address correspondence to Elizabeth J. Harry, elizabeth.harry@uts.edu.au.

* Present address: Shirin Ansari, EMBL Australia Node in Single Molecule Science and ARC Centre of Excellence in Advanced Molecular Imaging, School of Medical Sciences, University of New South Wales, Sydney, Australia.

Received 19 November 2020 Accepted 26 February 2021

Accepted manuscript posted online 15 March 2021

Published 7 May 2021 
of $Z$ rings at the cell poles, and the nucleoid occlusion protein, SImA, which inhibits $Z$ ring formation over unsegregated DNA $(4,5)$.

In addition to ensuring correct timing and positioning of the division site, there are numerous examples that demonstrate that the inhibition of division is equally important for cell survival under conditions such as DNA damage, protection from predation, progression of infection, and pathogenesis (6-8). Inhibition of division results in the formation of filamentous cells, a process where cell growth and DNA replication continue in the absence of division, resulting in elongated cells (6). Filamentation is an important survival mechanism utilized by several bacteria in response to environmental stimuli $(6,8)$.

A well-characterized cellular pathway that leads to filamentation is the SOS response, which is activated by DNA damage under conditions including oxidative stress, antibiotic treatment, or UV exposure (9). The activation of the SOS response is coordinated by two regulatory proteins, RecA and LexA $(10,11)$. RecA binds to singlestranded DNA (ssDNA) breaks caused by DNA damage, forming an RecA-DNA filament, which facilitates the self-cleavage of the LexA repressor and the subsequent upregulation (derepression) of LexA-controlled genes (12). LexA represses over 40 genes in $E$. coli under normal growth conditions (13).

A key function of the SOS response is to inhibit cell division. This is thought to allow sufficient time for DNA repair to occur before committing to producing the next generation of daughter cells, minimizing the transmission of defective DNA (14). In E. coli, this is facilitated by the cell division inhibitor, SulA, which is under the regulatory control of LexA (9). SulA is perhaps one of the most studied cell division inhibitors that causes filamentation, with molecular studies showing that it directly interacts with FtsZ, preventing assembly of the Z ring (15-19). When DNA damage is repaired, SulA is degraded via the cytoplasmic protease, Lon, and FtsZ polymerization and cell division resume $(20,21)$.

While SulA is generally the only cell division inhibitor commonly attributed to cell division inhibition during the SOS response in $E$. coli, other cell division inhibitors have been identified in this organism. Interestingly, several of these inhibitors include genes carried within prophages (22-26). The e14 prophage contains an unidentified SOS-inducible cell division inhibitor, previously named SfiC, which has been shown to contribute to filamentation during the SOS response, independent of SulA $(27,28)$. This inhibitor was not, however, under LexA repression (27). Phages contain their own repressor systems, which are LexA-like in nature, such as the $\mathrm{Cl}$ repressor from $\lambda$ phage $(13,29)$. The gene cohE from e14 encodes a Cl-like repressor, similar in sequence to other bacteriophage $\mathrm{Cl}$ repressors that are responsive to an SOS signal (30). As such, it is possible that expression of sfiC is regulated by cohE. In earlier work, it was also shown that FtsZ was most likely the target of SfiC, as point mutations in $\mathrm{fts} Z$ that confer resistance to the inhibitory effects of SulA also conferred resistance to $\operatorname{SfiC}(27,28)$. Based on prophage arrangement, the gene responsible for the SfiC phenotype has been suggested to be likely encoded by either of the adjacent e14 prophage genes ymfL or ymfM (30); however, the precise identity of the gene remains unknown.

We previously developed a high-throughput flow cytometry system to screen a novel E. coli expression library for candidate cell division regulators (31). We identified a DNA fragment containing the e14 prophage-carried genes, the full ymfM gene, and a partial sequence of adjacent genes, ymfL and oweE (previously annotated as ymfN), that when expressed in an inducible plasmid-based system caused cells to elongate and form filaments $(31,32)$. Since this DNA fragment contained both potential candidates for SfiC, ymfM and ymfL, further work was needed to determine which of these genes is responsible for the SfiC phenotype, i.e., SulA-independent filamentation during the SOS response.

Here, we show that ymfM is responsible for the SfiC phenotype, resulting in the inhibition of cell division when expressed from an inducible plasmid. We further characterize the effect of $y m f M$ expression on $E$. coli cell division and show that it results in prevention of the early 
A

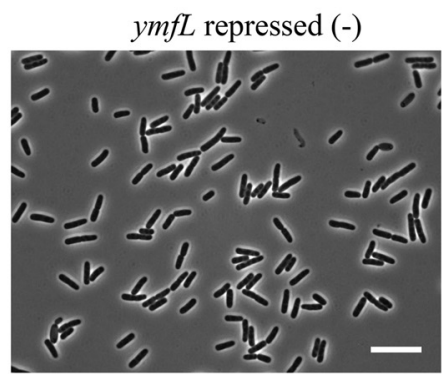

B

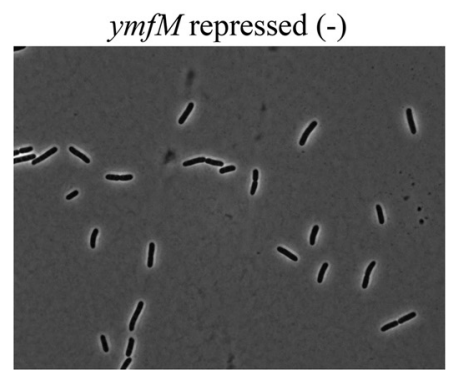

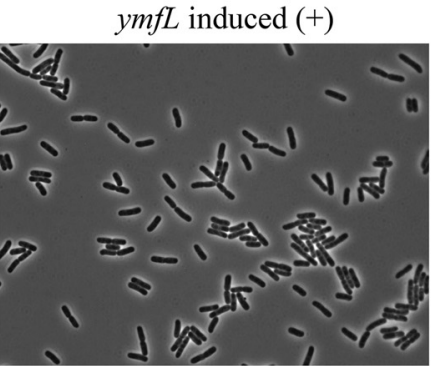

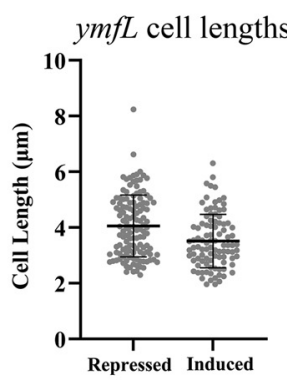

ymf $M$ induced (+)

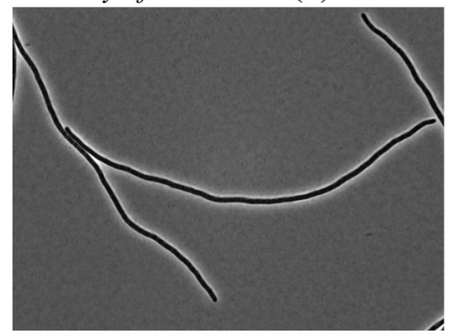

$y m f M$ cell lengths



FIG 1 Cell length distribution when ymfM or $y m f L$ is expressed from an inducible plasmid. The open reading frame for genes ymfL (A) and ymfM (B) were cloned into the arabinose-inducible plasmid, pBAD24, and grown in LB medium supplemented with $0.2 \%$ (wt/vol) glucose to repress their expression or $0.2 \%$ (wt/vol) arabinose to induce expression for $2 \mathrm{~h}$ (4 generations). Representative phase-contrast image of induced ymfM expression shows filamentation of cells, while the expression of $y m f L$ does not affect cell length. Approximately 100 cells were measured for each population, and cells lengths are shown on a scatterplot (error bars denote mean \pm standard deviation [SD]) on the right. Scale bar for all images is $10 \mu \mathrm{m}$.

stage of cell division, $Z$ ring formation. Furthermore, its inhibition pathway is independent of known cell division inhibitors SulA, SImA, and MinC. Finally, we show that YmfM causes filamentation during activation of the SOS response and that this is independent of SulA. Our data indicate that multiple division inhibitors exist during the SOS response and raise questions regarding their purpose for the survival of $E$. coli during times of stress.

\section{RESULTS}

Identification of a new gene, ymfM, whose expression induces filamentation. Previous independent theoretical $(30)$ and experimental $(27,31)$ studies have identified that either $y m f M$ or $y m f L$ is sfiC. To determine which one of these genes is responsible for the SfiC filamentous phenotype, ymfM and ymfL were cloned separately into the arabinose-inducible plasmid, pBAD24. Cells were grown in LB supplemented with $0.2 \%$ glucose to mid-exponential phase to repress gene expression. The cells were then diluted in fresh LB supplemented with $0.2 \%$ arabinose to induce gene expression and then grown for at least 4 doubling times (generation time is approximately $30 \mathrm{~min}$ ) to allow for enough time to observe cell length changes. The degree of filamentation associated with the expression of each individual gene was measured as cell length $(\mu \mathrm{m})$ from phase-contrast microscopy images. Control cells expressing just pBAD24 had an average cell length of $4.2 \pm 1.5 \mu \mathrm{m}$, and these ranged from approximately $2 \mu \mathrm{m}$ to $10 \mu \mathrm{m}$ in length (see Fig. S1 in the supplemental material). Therefore, filamentous cells resulting from gene expression in pBAD24 were defined as being greater than $10 \mu \mathrm{m}$ in cell length.

The induction of $y m f L$ expression did not cause filamentation, with cells having an average length of $3.5 \pm 0.9 \mu \mathrm{m}$ (Fig. 1A). However, expression of $y m f M$ resulted in inhibition of division, giving rise to an exclusively filamentous population having an average cell length of $57.3 \pm 19.7 \mu \mathrm{m}$ (Fig. 1B). In the repressed state, cells had the same cell-length distribution as the empty vector (see Fig. S1 in the supplemental material). The initial doubling time was $30 \mathrm{~min}$, which for the 120-min total incubation would result in filamentous cells 
16-fold (24) longer than their short-cell counterpart. The mean length of $y m f M$-induced cells was 14.5 times the $y m f M$-repressed mean of $3.9 \mu \mathrm{m}$. Overall, these results identify conclusively that $y m f M$ is the gene responsible for the filamentation observed in the $E$. coli overexpression screen (31) and in the SfiC phenotype (27).

ymfM expression inhibits the earliest stage of division: FtsZ ring formation. To understand whether induction of ymfM expression inhibits cell division by impeding FtsZ assembly or a later stage of cell division, immunofluorescence microscopy (IFM) was used to measure $Z$ ring frequency in cells induced to express $y m f M$ from pBAD24. The absence of $Z$ rings in the $y m f M$-induced cells would imply that $Z$ ring formation has been inhibited.

IFM using cephalexin-treated filamentous cells was first performed as a control to show that the technique did not affect the integrity of the $Z$ rings in filaments, and other control experiments showed that the antibody detection for IFM is specific for FtsZ (see Fig. S2 in the supplemental material).

Next, wild-type cells harboring pBAD-ymfM were grown in LB for 3 generations with either glucose to repress expression or with arabinose to induce expression for $90 \mathrm{~min}$. During repressed conditions, cells had an average cell length of $3.5 \pm 0.9 \mu \mathrm{m}$, indicating that they were dividing at the normal frequency. In these short cells, $Z$ rings were observed as bright green bands at midcell (Fig. 2A, white arrow). The number of $Z$ rings was scored from visual inspection of approximately 100 cells. For each cell, the cell length was also measured from the image. The frequency of $Z$ ring observation was calculated by dividing the total length of all cells counted by the total number of $Z$ rings observed (micrometers $/ Z$ ring). In the short cell population, $Z$ rings were observed at a frequency of $8.4 \mu \mathrm{m} / Z$ ring. In cells expressing $y m f M$, the average cell length was $35.5 \pm 23.1 \mu \mathrm{m}$, and almost no $Z$ rings were observed along the length of the filament. Instead, FtsZ appeared to be diffused throughout the filament (Fig. 2B). Occasional $Z$ rings were seen in this sample; however, these were primarily in the few short cells present. $Z$ rings in this population were observed at a frequency of $224 \mu \mathrm{m} /$ $Z$ ring-27-fold less frequently than repressed cells. DAPI (4',6-diamidino-2-phenylindole) (DNA) staining showed that in $y m f M$-induced filaments the nucleoids appeared normal, suggesting that the filamentation observed is solely due to the inhibition of division and not a result of inhibition of DNA replication or chromosome segregation. Furthermore, it was shown that the lack of $Z$ rings in filaments was not due to changes in cellular levels of FtsZ (see Fig. S3 in the supplemental material), indicating that YmfM does not prevent $Z$ ring formation by proteolysis or degradation of FtsZ.

In summary, induced expression of $y m f M$ results in inhibition of $Z$ ring formation at midcell, and this inhibition is likely affecting FtsZ mechanistically as cellular levels of FtsZ are not affected.

YmfM inhibition of division does not rely on known cell division regulators, SulA, SImA, and MinC. Induced expression of ymfM inhibits the earliest stage of division, as $Z$ rings were not detected with IFM (Fig. 2). We next tested whether this inhibition occurred through other $Z$ ring regulators. These include SulA, which inhibits Fts $Z$ from assembling into a ring during the SOS response $(15,16)$; MinC, which prevents FtsZ assembly at cell poles under normal conditions $(33,34)$; and SImA, which prevents FtsZ assembly over nucleoids as part of the nucleoid occlusion system in $\operatorname{E}$. coli $(35,36)$. The expression of ymfM was induced in $E$. coli cells in the absence of SulA, ( $\Delta$ sulA; JW0941) (37), SImA ( $\Delta \operatorname{simA}$; JW5641) (37), or the Min system ( $\Delta \min C D E ;$ TB43) (35). If YmfM prevents $Z$ ring assembly specifically via any one of these other inhibitors, then no filamentation will be observed in their absence when ymfM is expressed.

When ymfM expression was repressed, the mutant strains had a short cell distribution, which was comparable to their wild-type counterpart, except for the $\triangle \min C D E$ strain. The absence of the Min proteins is reported to cause a mixed population of minicells (small cells lacking DNA) and mildly filamentous cells due to polar cell division (15), and this was observed in the $\triangle \min C D E$ strain cell population. Cell lengths were $3.9 \pm 1.2 \mu \mathrm{m}$ (BW25113, parent of the $\Delta s u l A$ and $\Delta s / m A$ strains), $3.8 \pm 1.0 \mu \mathrm{m}$ ( $\Delta s u l A$ 
A


B
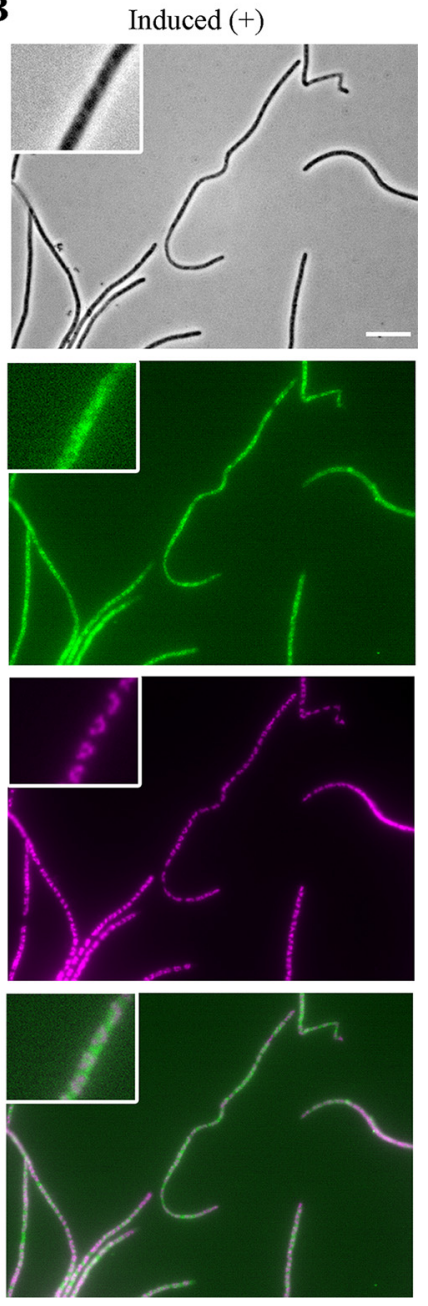

FIG 2 Z ring assembly is inhibited in filamentous cells induced by ymfM expression. Immunofluorescence microscopy using anti-FtsZ antiserum to visualize $Z$ rings of strain BW25113 carrying pBAD-ymfM. (A) Cells grown in LB supplemented with $0.2 \%$ (wt/vol) glucose to repress ymfM expression short cells are present with $Z$ rings at midcell (arrows in inset indicate $Z$ ring present as green bands). (B) Expression of ymfM was induced with $0.2 \%$ (wt/vol) arabinose for three generations $(90 \mathrm{~min})$, and the resulting filamentous cells contain no Z rings. The nucleoids have been stained with DAPI (falsely colored magenta), and the overlay image shows $Z$ ring positioning within cells relative to nucleoids. Scale bars for all images are $10 \mu \mathrm{m}$.

strain), $5.7 \pm 4.2 \mu \mathrm{m}$ ( $\Delta$ s/mA strain), $4.3 \pm 1.4 \mu \mathrm{m}$ (TB28, parent of the $\Delta \min C D E$ strain), and $10.6 \pm 5.5 \mu \mathrm{m}(\triangle \min C D E$ strain).

When ymfM expression was induced in the mutant strains, $\Delta$ sulA strain, $\Delta \operatorname{sim} A$ strain, or $\triangle \min C D E$ strain, filamentation was observed (Fig. 3). The average cell lengths were $71.8 \pm 19.7 \mu \mathrm{m}$ ( $\Delta$ sulA strain), $70.6 \pm 28.3 \mu \mathrm{m}(\Delta \mathrm{s} / \mathrm{mA}$ strain), and $87.7 \pm 16.1 \mu \mathrm{m}$ ( $\triangle \min C D E$ strain), respectively. Filamentation in the mutant strains was comparable to that of their respective wild-type parent strain (Fig. 3B), with average cell lengths of $76.2 \pm 10.8 \mu \mathrm{m}$ for BW25113 and $93.9 \pm 15.4 \mu \mathrm{m}$ for TB28. The results here show that ymfM does not require the inhibitory activity of SulA, SImA, or the Min system to inhibit $Z$ ring formation.

YmfM is involved in the inhibition of division during the SOS response and is independent of SulA. Several years ago, an unknown gene, sfiC, was identified as a cell division inhibitor during the SOS response (27). D'Ari and Huisman (27) found that even in the absence of the sulA gene, cells were able to filament when SOS was activated, as sfiC would inhibit cell division. We have thus far shown that ymfM is sfiC, as its expression from an inducible plasmid causes filamentation (Fig. 1), and this is independent of the 
A BW25113

JW0941

JW5641

TB28

(Wild-type)

TB43

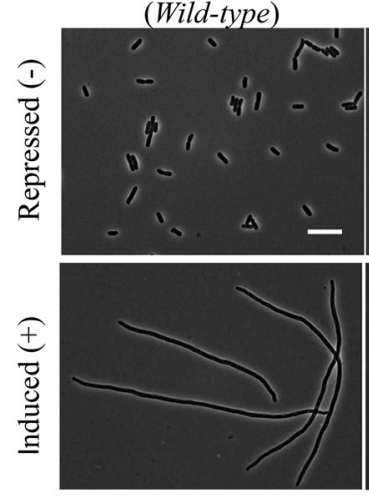

(4sulA)

$(\Delta \operatorname{sim} A)$

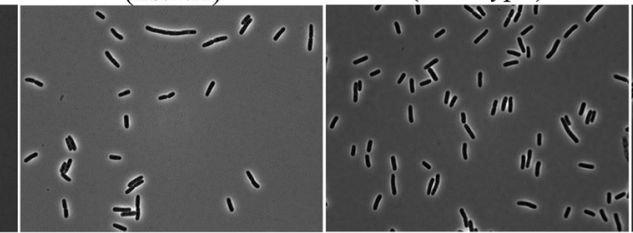

$(\triangle \min C D E)$


B i)

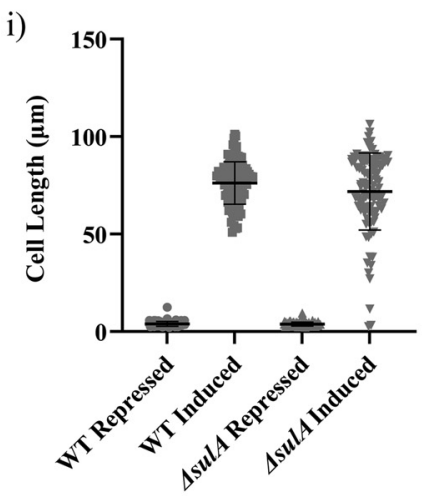

ii)

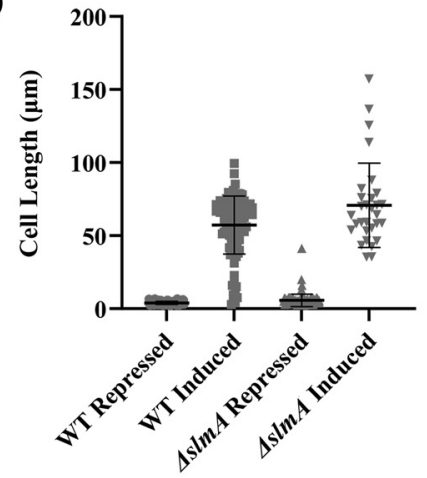

iii)

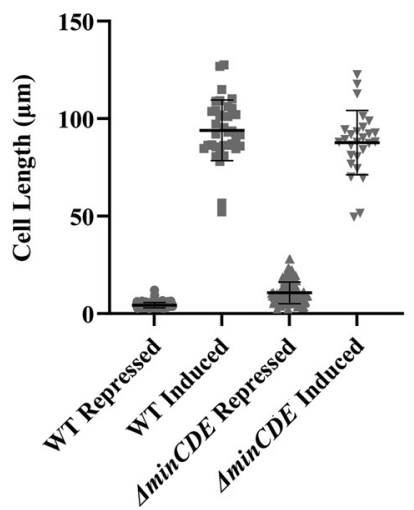

FIG 3 Filamentation caused by the expression of $y m f M$ is independent of the cell division inhibitors SulA, SImA, and the Min system. (A) Phase-contrast images of $\Delta$ sulA (JW0941), $\Delta$ s/mA (JW5641), and $\Delta \operatorname{minCDE}$ (TB43) strains and their wild-type backgrounds (BW25113 and TB28, respectively) show filamentation when ymfM expression is induced from pBAD24 with $0.2 \%$ (wt/vol) arabinose in LB for $2 \mathrm{~h}$ (4 generations). Short cells are observed when ymfM expression is repressed with $0.2 \%$ (wt/vol) glucose, with the exception of the $\Delta m i n C D E$ strain, which has a mixed population of short, slightly filamentous, and minicells due to increased division at cell poles. (B) Cell length scatterplots of the mutants, $\Delta$ sulA strain (i), $\Delta$ s/mA strain (ii), and $\triangle$ minCDE strain (iii), show that the degree of filamentation caused by $y m f M$ expression is comparable to that of their wild-type counterparts. Approximately 100 cells were measured for each population and cells lengths are shown on a scatterplot (error bars denote mean $\pm \mathrm{SD}$ ) on the right. Scale bar for all images is $10 \mu \mathrm{m}$.

inhibitory actions of SulA (Fig. 3). ymfM therefore could also be responsible for the SulA-independent filamentation observed during the SOS response.

To show $y m f M$ was required for filamentation during SOS in the absence of sulA, we used an approach similar to that of D'Ari and Huisman (27), in which sfiC was identified using a temperature-sensitive mutant, recA441, also known as recA-tif (38). In this mutant, at the nonpermissive temperature of $42^{\circ} \mathrm{C}$, constitutive protease activity of RecA is observed, thereby inducing the SOS response and filamentation without the need for external means to DNA damage (39). We, therefore, cloned recA441 into pBAD24. Gene expression and subsequent SOS was induced with $0.2 \%$ arabinose and growth at $42^{\circ} \mathrm{C}$ in the $\triangle y m f M, \Delta$ sulA, or $\Delta$ sulA $\triangle y m f M$ strains. Any changes to the degree of filamentation were measured. If, in the absence of $y \mathrm{mfM}$, cells do not filament as effectively as their wild-type counterparts, this would suggest that $y m f M$ directly contributes to filamentation during the SOS response.

As expected, induction of recA441 caused filamentation in the wild-type background (Fig. $4 A$ and B, green; Fig. $4 C$, i) compared to that of the short cell population of wild-type cells expressing empty vector (Fig. $4 A$ and B, blue). Here, cell size (reported as $\mu \mathrm{m}^{3}$ ) was measured using a Coulter cytometer, and this is proportional to cell length as cell width is unchanged.

There was a reduction in the degree of filamentation in the absence of sulA alone (Fig. 4 A and B, red; Fig. 4C, ii) compared to that of its wild-type counterpart. However, 
A

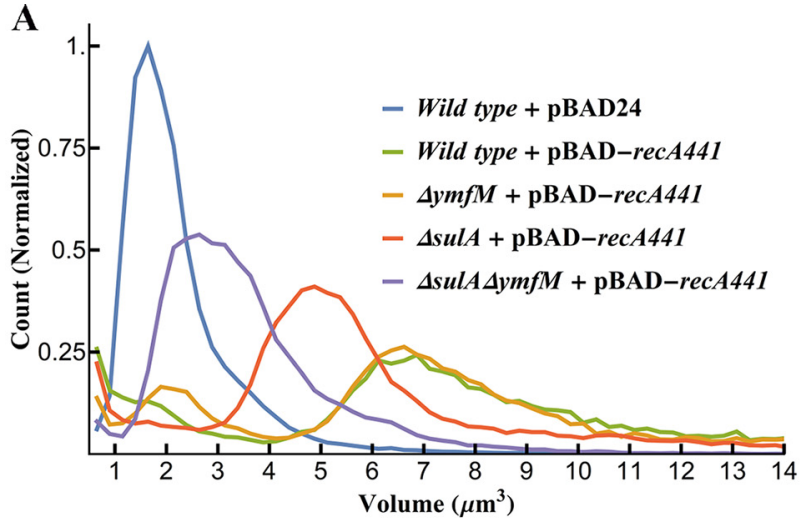

B



\section{C}

i)

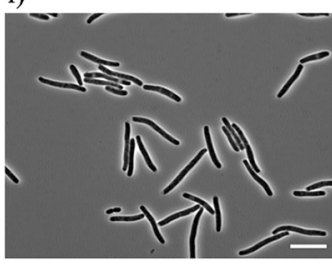

ii)



iv)

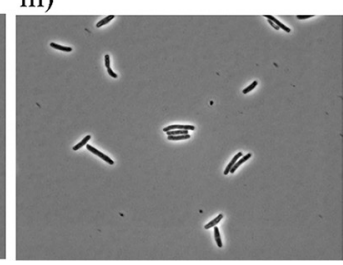

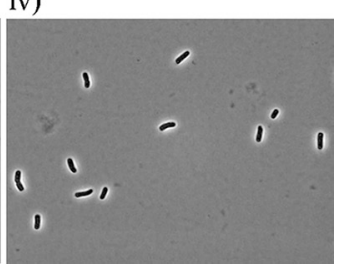

FIG $4 \mathrm{YmfM}$ contributes to the filamentation of $E$. coli during induction of the SOS response using the temperaturesensitive RecA mutant, recA441. (A) Coulter cytometer analysis of cell size distribution of wild type, $\triangle y m f M$ strain, $\triangle$ sulA strain, and $\triangle$ sulA $\triangle y m f M$ strain after $2 \mathrm{~h}$ of pBAD-recA441 induction in LB with $0.2 \%$ (wt/vol) arabinose and $100 \mu \mathrm{g} / \mathrm{ml}$ adenine at $42^{\circ} \mathrm{C}$. Samples were compared to wild-type cells expressing empty pBAD24 (blue) not under SOS induction. The $x$ axis represents cell volume, and the $y$ axis represents cell count which has been normalized to 1 . Pulse data are greater than 10,000 events (cells). Data represent one biological replicate. Three additional biological repeats were performed (see Fig. S4 in the supplemental material). (B) The degree of filamentation of the mutant strains relative to wild-type filaments. The data from four biological replicates have been normalized so that mode of wild-type pBAD24 (short cells) is 0\% and mode of wild-type pBAD-recA441 (filaments) is 100\%. Error bars are mean \pm SD. (C) Representative phase-contrast image of wild type (i), $\triangle$ sulA strain (ii), $\triangle y m f M$ strain (iii), and $\triangle$ sulA $\triangle y m f M$ strain (iv), all expressing pBAD-recA441. Scale bar for all images is $10 \mu \mathrm{m}$.

this did not fully recover to the short cell population (Fig. 4A and B, blue), highlighting the presence of an additional cell division inhibitor(s) that is active during the SOS response. The absence of $y m f M$ (Fig. $4 \mathrm{~A}$ and B, yellow; Fig. $4 \mathrm{C}$, iii) was similar to that of induction of recA441 in wild-type cells (Fig. $4 A$ and $B$, green), so the absence of $y m f M$ alone does not result in less filamentation in the population. However, in the absence of both sulA and ymfM (Fig. 4A and B, purple; Fig. 4C, iv), there was the greatest shift toward the shorter cell population (blue line). The difference in cell size distribution of $\triangle$ sulA $\triangle y m f M$ strain (purple) cells compared to $\Delta$ sulA strain (red) cells shows that $y m f M$ does indeed play a role in inhibiting division during the SOS response when recA441 is induced and this role becomes evident when SulA is absent. Importantly, while the shift toward shorter cells was the greatest for the $\Delta$ sulA $\triangle y m f M$ strain (purple), it did not lead to a full recovery to a short cell population (blue), suggesting the presence of additional SOS-inducible inhibitors in this organism. These trends were observed in multiple biological replicates (represented in Fig. 4B; see also Fig. S4 in the supplemental material).

\section{DISCUSSION}

We have identified the gene, $y m f M$, which when expressed from an inducible plasmid causes arrest in cell division (Fig. 1). YmfM is encoded within the e14 prophage, which had been indicated to harbor the gene responsible for the SOS-inducible filamentation phenotype, SfiC $(27,28)$. The exact gene responsible for SfiC has remained elusive for over 35 years and was narrowed down to either ymfL or ymfM in 2004 (30). 
In this work, we eliminate $y m f L$ and confirm ymfM as being responsible for the inhibition of division, confirming that ymfM is responsible for the filamentous SfiC phenotype reported in 1983 (27).

We first characterized the stage of division being inhibited by $y m f M$. The expression of $y m f M$ inhibits $Z$ ring formation, as essentially no $Z$ rings were seen in cells expressing ymfM. This is consistent with previous work by D'Ari and Huisman (27), who demonstrated that mutations in $\mathrm{fts} Z$ that confer resistance to the inhibitory effects of SulA also confer resistance to SfiC. We also show that YmfM does not act through known $E$. coli cell division regulators SulA, SImA, or MinC. Another phage-encoded inhibitor, DicB, from prophage Qin has been reported to utilize the inhibitory actions of MinC, as no filamentation by DicB was observed in the absence of the Min system $(40,41)$. Filamentation by YmfM was observed in the absence of $\operatorname{sul} A, \operatorname{sim} A$, and $\min C D E$, showing that it is independent of these inhibitory pathways. It remains to be seen whether ymfM targets FtsZ directly to inhibit Z ring assembly or if it does so through early division proteins such as FtsA or ZipA (3), similar to the phage inhibitor Kil $(22,42)$. Our data do not rule out the possibility that YmfM causes division inhibition indirectly through an as yet unidentified cell division inhibitor.

Since we have shown that induction of $y m f M$ expression causes filamentation and is responsible for the SfiC phenotype, then, as an SOS-inducible gene, it should also contribute to filamentation when the SOS response is induced through the activation of RecA, as reported by D'Ari and Huisman (27). This indeed was the case. Through activation of recA441, YmfM inhibited division during the SOS response (Fig. 4). This was apparent when the degree of filamentation was compared between the $\Delta$ sulA and $\Delta s u l A$ $\Delta y m f M$ strains, as the double knockout resulted in shorter filaments compared to the $\triangle$ sulA strain alone. This observed difference in filamentation can be attributed to $y m f M$.

As the double-knockout strain showed that $y m f M$ is contributing to the filamentation seen in this assay, we expected that, similar to the $\Delta$ sulA strain, the absence of ymfM alone ( $\triangle y m f M)$ would also result in a slightly shorter cell population compared to that of the wild type (Fig. 4). However, this was not the case and was unexpected given that induced expression of ymfM from pBAD-ymfM results in a strong filamentous phenotype (Fig. 1B). This could be due to differences in expression levels of ymfM from the plasmid versus through SOS induction. Furthermore, it is likely that in the SOS-induced system, as SulA is still present in the $\triangle y m f M$ strain, SulA and potential additional cell division inhibitors are masking the phenotypic effects that can be caused by the absence of ymfM.

It was also apparent that genes additional to sulA and ymfM contribute to filamentation during RecA-activated SOS, as there was not a full recovery to a short-cell population in the $\Delta$ sulA $\Delta y m f M$ strain background (Fig. 4B). Given that over 1,000 genes are differentially expressed during the SOS response (43), it is likely that several SOS-inducible cell division inhibitors are yet to be identified. For example, KilR, another prophage-encoded cell division inhibitor, has only recently been shown to be activated by the small RNA, oxyS, in response to oxidative stress (44).

It is interesting to speculate why multiple cell division inhibitors are present during the SOS response and ask the following question: how may they differ to SulA? Additionally, why are so many of these division inhibitors present in prophages? It has been shown that the 9 cryptic prophages present in E. coli K-12 are beneficial for survival and adaption under different environmental conditions and signals, including osmotic, oxidative and acid stress, biofilm formation, and tolerance to antibiotics $(28,30$, 45). Several of these prophages also encode cell division inhibitors, such as Kil, DicB, DicF, and CbtA (23-26). kilR from prophage Rac and dicB from prophage Qin are both cell division inhibitors that have been shown to be upregulated during treatment with nalidixic acid and azlocillin and are thought to contribute to the resistance of these antibiotics $(24,25,45)$. It is possible that these prophage-carried inhibitors, originally serving the functions of the phage, have more recently been adapted to be induced in response to specific environmental cues for the benefit of the bacterium $(45,46)$. 
It was of interest to us to understand conditions under which ymfM is active, as this may help us differentiate how its function differs from that of SulA. Expression of ymfM is upregulated during norfloxacin-induced SOS (47-49). However, while we could also show that ymfM expression was upregulated under these conditions, we were repeatedly unable to show the requirement for this gene in causing filamentation in the presence of this antibiotic (data not shown) as we observed in the RecA441 experiments (Fig. 4). As with the recA441 experiments, we think it likely that the ymfM phenotype in the presence of norfloxacin is being masked by numerous filamentation mechanisms. Alternatively, since ymfM is not under the control of LexA repression (27), and is likely controlled by the $\mathrm{Cl}$-like repressor within the e14 prophage, it is possible that ymfM responds to different yet-to-be identified conditions. Like LexA, Cl repressors are responsive to SOS signals and are dependent on RecA-ssDNA binding to undergo autocleavage, resulting in expression of $\mathrm{Cl}$-repressed genes.

These observations also highlight the possibility that multiple cell division inhibitor genes and pathways exist for a more finely tuned division regulation response to the environment. For example, spatial inhibitors MinC and SImA have specialized functions to ensure that division does not occur at inappropriate times in the cell cycle or the incorrect position in the cell. It is probable that temporal inhibitors are equally specialized with respect to when they are activated. By having multiple inhibitors with subtly different properties, cells are likely able to tailor their inhibition in response to different environmental conditions and signals to maximize their survivability. More work is needed to tease apart the relationship between different inhibitors and the conditions under which they are required.

Overall, our data show that ymfM is a novel gene required for cell division inhibition during the SOS response, and its activity is independent of a major SOS-induced cell division inhibitor, SulA. Our data also highlight that our current understanding of the cell division regulation during the SOS response is incomplete and raises many questions. In particular, what are the benefits of having multiple cell division inhibitors during the SOS response? How are the different inhibitors activated? And, how do these multiple pathways help $E$. coli cope with stresses and aid in the survival of the population, if at all?

\section{MATERIALS AND METHODS}

Strains and growth conditions. All E. coli strains used in this study are listed in Table $\mathrm{S} 1$ in the supplemental material. E. coli cells were grown in LB medium with vigorous shaking $(250 \mathrm{rpm})$ at $37^{\circ} \mathrm{C}$ unless stated otherwise. Ampicillin ( $100 \mu \mathrm{g} / \mathrm{ml}$; Sigma-Aldrich) was supplemented where appropriate. Lambda red recombination $(37,50)$ was used to generate gene deletions in E. coli strains listed in Table S1.

Plasmid construction and expression. All plasmids used in this study are listed in Table S2 in the supplemental material. Recombinant plasmids were constructed using the Gibson assembly method (51) following the manufacturer's instructions for the Gibson assembly master mix (NEB). DNA fragments ymfM, ymfL, and oweE were amplified from BW25113 genomic DNA, and recA441 was amplified from JM12 genomic DNA (38) (see Table S3 in the supplemental material). Each DNA fragment contained homologous overlap to pBAD24, which was linearized at the Ncol restriction site.

Expression of DNA fragments from pBAD24. Cultures of desired $E$. coli strain containing recombinant pBAD24 were grown overnight in $5 \mathrm{ml} \mathrm{LB}$ with ampicillin $(100 \mu \mathrm{g} / \mathrm{ml})$ and $0.2 \%$ glucose (repression of araBAD promoter) at $37^{\circ} \mathrm{C}$ or $30^{\circ} \mathrm{C}$ for pBAD-recA441. Cultures were diluted to an optical density at $600 \mathrm{~nm}\left(\mathrm{OD}_{600}\right)$ of $0.04 \mathrm{in} 20 \mathrm{ml} \mathrm{LB}$ with ampicillin $(100 \mu \mathrm{g} / \mathrm{ml})$ and $0.2 \%$ glucose and grown at $37^{\circ} \mathrm{C}$ (or $30^{\circ} \mathrm{C}$ for pBAD-recA441), $250 \mathrm{rpm}$ to mid-exponential phase $\left(\mathrm{OD}_{600}\right.$ of $\left.\sim 0.5\right)$. A 1 - $\mathrm{ml}$ aliquot of the culture was collected and fixed with $3 \%$ formaldehyde. The remainder of the culture was pelleted by centrifugation at 2,000 $\times g$ and washed twice in an equal volume of fresh LB to remove the glucose. Cultures were further diluted to an $\mathrm{OD}_{600}$ of 0.04 in $20 \mathrm{ml} \mathrm{LB}$ with ampicillin $(100 \mu \mathrm{g} / \mathrm{ml})$ and $0.2 \%$ arabinose (to induce expression) and grown for 4 generations (approximately $2 \mathrm{~h}$ ) at $37^{\circ} \mathrm{C}$ with shaking. For recA441 expression, cultures were grown at $42^{\circ} \mathrm{C}$ and supplemented with $100 \mu \mathrm{g} / \mathrm{ml}$ adenine in addition to the arabinose and ampicillin.

Microscopy. (i) Immunofluorescence microscopy. IFM was used for the detection of the FtsZ protein and is based on the method previously described in reference 52, with the exception that cell lysis was omitted. Cultures of BW25113 containing PBAD-ymfM were grown as detailed above and incubated with the primary antibody anti-FtsZ (antisera) diluted 1:10,000 in bovine serum albumin (BSA)-phosphate-buffered saline (PBS) at $4^{\circ} \mathrm{C}$ overnight. The primary antibody was removed, and the cells were incubated with Alexa Fluor 488-conjugated secondary antibody, anti-rabbit IgG (Invitrogen), diluted $1: 10,000$ in BSA-PBS for $2 \mathrm{~h}$ in the dark at room temperature. Wells were washed with PBS to remove 
excess secondary antibody, and DAPI (4',6-diamidino-2-phenylindole) at a final concentration of $2 \mu \mathrm{g} / \mathrm{ml}$ was added to each sample. Cell morphology and FtsZ localization were then examined using phase-contrast and fluorescence microscopy as described below.

(ii) Phase-contrast and fluorescence microscopy. Cells were imaged using phase-contrast and fluorescence on a Zeiss Axioplan 2 fluorescence microscope equipped with a plan apochromat (100× numerical aperture [NA] 1.4; Zeiss) objective lens. The light source was a $100 \mathrm{~W}$ high-pressure mercury lamp passed through the following filter blocks for visualizing Alexa Fluor 488 (filter set 09, Zeiss; 450- to 490$\mathrm{nm}$ BP excitation filter, $515 \mathrm{~nm}$ long pass [LP] barrier filter) and for visualizing DAPI (filter set 02, Zeiss; 365$\mathrm{nm}$ excitation filter, $420 \mathrm{~nm}$ long pass [LP] barrier filter). Images were collected using the AxioCam MRm camera and processed using the AxioVision 4.8 software (Zeiss). Approximately 100 cells were measured from each data set (unless specified otherwise) using the length tool within the AxioVision software.

Coulter cytometer. A volume of $100 \mu \mathrm{l}$ of fixed cells was added to $9.9 \mathrm{ml}$ of IsoFlow buffer (Beckman). Of this, $200 \mu \mathrm{l}$ was run through a 50- $\mu \mathrm{m}$ aperture tube, and data were collected over 400 bins ranging from $0.6 \mu \mathrm{m}^{3}$ to $100 \mu \mathrm{m}^{3}$. Data were exported in excel and plotted as a histogram with the cell volume along the $x$ axis and normalized cell counts on the $y$ axis in Mathematica (Wolfram). To compare the shift in cell size across all four biological replicates, the mode of each strain from all replicates was calculated. The mode of wild type expressing pBAD24 (short cells) was set to 0\% filamentation, and the mode of wild type expressing pBAD-recA441 (filaments) was set to $100 \%$ filamentation to normalize all values. The modes of each mutant strain across all four biological replicates were then combined to show the relative shift in filamentation between strains.

\section{SUPPLEMENTAL MATERIAL}

Supplemental material is available online only. SUPPLEMENTAL FILE 1, PDF file, 2 MB.

\section{ACKNOWLEDGMENT}

This work was partly supported by an Australian Government Research Training Program Stipend Scholarship awarded to Shirin Ansari.

\section{REFERENCES}

1. Harry E, Monahan L, Thompson L. 2006. Bacterial cell division: the mechanism and its precison. Int Rev Cytol 253:27-94. https://doi.org/10.1016/ S0074-7696(06)53002-5.

2. Haeusser DP, Margolin W. 2016. Splitsville: structural and functional insights into the dynamic bacterial Z ring. Nat Rev Microbiol 14:305-319. https://doi.org/10.1038/nrmicro.2016.26.

3. den Blaauwen T, Hamoen LW, Levin PA. 2017. The divisome at 25: the road ahead. Curr Opin Microbiol 36:85-94. https://doi.org/10.1016/j.mib .2017.01.007.

4. Adams DW, Wu LJ, Errington J. 2014. Cell cycle regulation by the bacterial nucleoid. Curr Opin Microbiol 22:94-101. https://doi.org/10.1016/j.mib 2014.09.020.

5. Rowlett VW, Margolin W. 2015. The Min system and other nucleoid-independent regulators of $Z$ ring positioning. Front Microbiol 6:478. https:// doi.org/10.3389/fmicb.2015.00478.

6. Justice SS, Hunstad DA, Cegelski L, Hultgren SJ. 2008. Morphological plasticity as a bacterial survival strategy. Nat Rev Microbiol 6:162-168. https:// doi.org/10.1038/nrmicro1820.

7. Justice SS, Harrison A, Becknell B, Mason KM. 2014. Bacterial differentiation, development, and disease: mechanisms for survival. FEMS Microbiol Lett 360:1-8. https://doi.org/10.1111/1574-6968.12602.

8. Yang DC, Blair KM, Salama NR. 2016. Staying in shape: the impact of cell shape on bacterial survival in diverse environments. Microbiol Mol Biol Rev 80:187-203. https://doi.org/10.1128/MMBR.00031-15.

9. Simmons LA, Foti JJ, Cohen SE, Walker GC. 25 July 2008. The SOS regulatory network. EcoSal Plus 2008. https://doi.org/10.1128/ecosalplus.5.4.3.

10. Little JW. 1991. Mechanism of specific LexA cleavage: autodigestion and the role of RecA coprotease. Biochimie 73:411-421. https://doi.org/10 .1016/0300-9084(91)90108-d.

11. Little JW, Gellert M. 1983. The SOS regulatory system: control of its state by the level of RecA protease. J Mol Biol 167:791-808. https://doi.org/10 .1016/s0022-2836(83)80111-9.

12. Janion C. 2008. Inducible SOS response system of DNA repair and mutagenesis in Escherichia coli. Int J Biol Sci 4:338-344. https://doi.org/10 .7150/ijbs.4.338.

13. Butala M, Zgur-Bertok D, Busby SJ. 2009. The bacterial LexA transcriptional repressor. Cell Mol Life Sci 66:82-93. https://doi.org/10.1007/ s00018-008-8378-6.
14. Huisman O, D'Ari R, Gottesman S. 1984. Cell-division control in Escherichia coli: specific induction of the SOS function SfiA protein is sufficient to block septation. Proc Natl Acad Sci U S A 81:4490-4494. https://doi .org/10.1073/pnas.81.14.4490.

15. Bi E, Lutkenhaus J. 1993. Cell division inhibitors SulA and MinCD prevent formation of the FtsZ ring. J Bacteriol 175:1118-1125. https://doi.org/10 $.1128 /$ jb.175.4.1118-1125.1993.

16. Cordell SC, Robinson EJ, Lowe J. 2003. Crystal structure of the SOS cell division inhibitor SulA and in complex with FtsZ. Proc Natl Acad Sci U S A 100:7889-7894. https://doi.org/10.1073/pnas.1330742100.

17. Justice SS, Garcia-Lara J, Rothfield LI. 2000. Cell division inhibitors SulA and MinC/MinD block septum formation at different steps in the assembly of the Escherichia coli division machinery. Mol Microbiol 37:410-423. https://doi.org/10.1046/j.1365-2958.2000.02007.x.

18. Dajkovic A, Mukherjee A, Lutkenhaus J. 2008. Investigation of regulation of FtsZ assembly by SulA and development of a model for FtsZ polymerization. J Bacteriol 190:2513-2526. https://doi.org/10.1128/JB.01612-07.

19. Chen Y, Milam SL, Erickson HP. 2012. SulA inhibits assembly of FtsZ by a simple sequestration mechanism. Biochemistry 51:3100-3109. https://doi .org/10.1021/bi201669d.

20. Justice SS, Hunstad DA, Seed PC, Hultgren SJ. 2006. Filamentation by Escherichia coli subverts innate defenses during urinary tract infection. Proc Natl Acad Sci U S A 103:19884-19889. https://doi.org/10.1073/pnas .0606329104 .

21. Mizusawa S, Gottesman S. 1983. Protein degradation in Escherichia coli: the lon gene controls the stability of sulA protein. Proc Natl Acad Sci U S A 80:358-362. https://doi.org/10.1073/pnas.80.2.358.

22. Haeusser DP, Hoashi M, Weaver A, Brown N, Pan J, Sawitzke JA, Thomason LC, Court DL, Margolin W. 2014. The Kil peptide of bacteriophage lambda blocks Escherichia coli cytokinesis via ZipA-dependent inhibition of FtsZ assembly. PLoS Genet 10:e1004217. https://doi.org/10 .1371/journal.pgen.1004217.

23. Heller DM, Tavag M, Hochschild A. 2017. CbtA toxin of Escherichia coli inhibits cell division and cell elongation via direct and independent interactions with FtsZ and MreB. PLoS Genet 13:e1007007. https://doi.org/10 .1371/journal.pgen.1007007.

24. Conter A, Bouche JP, Dassain M. 1996. Identification of a new inhibitor of essential division gene $\mathrm{fts} Z$ as the kil gene of defective prophage Rac. J 
Bacteriol 178:5100-5104. https://doi.org/10.1128/jb.178.17.5100-5104 1996.

25. Cam K, Bejar S, Gil D, Bouche JP. 1988. Identification and sequence of gene dicB: translation of the division inhibitor from an in-phase internal start. Nucleic Acids Res 16:6327-6338. https://doi.org/10.1093/nar/16.14 .6327.

26. Tan Q, Awano N, Inouye M. 2011. YeeV is an Escherichia coli toxin that inhibits cell division by targeting the cytoskeleton proteins, FtsZ and MreB. Mol Microbiol 79:109-118. https://doi.org/10.1111/j.1365-2958 .2010.07433.x.

27. D'Ari R, Huisman O. 1983. Novel mechanism of cell division inhibition associated with the SOS response in Escherichia coli. J Bacteriol 156:243-250. https://doi.org/10.1128/JB.156.1.243-250.1983.

28. Maguin E, Brody H, Hill CW, D'Ari R. 1986. SOS-associated division inhibition gene sfiC is part of excisable element e14 in Escherichia coli. J Bacteriol 168:464-466. https://doi.org/10.1128/jb.168.1.464-466.1986.

29. Galkin VE, Yu X, Bielnicki J, Ndjonka D, Bell CE, Egelman EH. 2009. Cleavage of bacteriophage lambda $\mathrm{cl}$ repressor involves the RecA C-terminal domain. J Mol Biol 385:779-787. https://doi.org/10.1016/j.jmb.2008.10 .081 .

30. Mehta P, Casjens S, Krishnaswamy S. 2004. Analysis of the lambdoid prophage element e14 in the E. coli K-12 genome. BMC Microbiol 4:4. https:// doi.org/10.1186/1471-2180-4-4.

31. Burke C, Liu M, Britton W, Triccas JA, Thomas T, Smith AL, Allen $S$, Salomon R, Harry E. 2013. Harnessing single cell sorting to identify cell division genes and regulators in bacteria. PLoS One 8:e60964. https://doi .org/10.1371/journal.pone.0060964.

32. Mediati DG, Burke CM, Ansari S, Harry EJ, Duggin IG. 2018. High-throughput sequencing of sorted expression libraries reveals inhibitors of bacterial cell division. BMC Genomics 19:781. https://doi.org/10.1186/s12864 -018-5187-7.

33. Hu Z, Lutkenhaus J. 1999. Topological regulation of cell division in Escherichia coli involves rapid pole to pole oscillation of the division inhibitor MinC under the control of MinD and MinE. Mol Microbiol 34:82-90. https://doi.org/10.1046/j.1365-2958.1999.01575.x.

34. Hu Z, Mukherjee A, Pichoff S, Lutkenhaus J. 1999. The MinC component of the division site selection system in Escherichia coli interacts with FtsZ to prevent polymerization. Proc Natl Acad Sci U S A 96:14819-14824. https://doi.org/10.1073/pnas.96.26.14819.

35. Bernhardt TG, de Boer PAJ. 2005. SImA, a nucleoid-associated, FtsZ binding protein required for blocking septal ring assembly over chromosomes in E. coli. Mol Cell 18:555-564. https://doi.org/10.1016/j.molcel.2005.04 .012 .

36. Schumacher MA, Zeng W. 2016. Structures of the nucleoid occlusion protein SImA bound to DNA and the C-terminal domain of the cytoskeletal protein FtsZ. Proc Natl Acad Sci U S A 113:4988-4993. https://doi.org/10 $.1073 /$ pnas. 1602327113.

37. Baba T, Ara T, Hasegawa M, Takai Y, Okumura Y, Baba M, Datsenko KA, Tomita M, Wanner BL, Mori H. 2006. Construction of Escherichia coli K-12 in-frame, single-gene knockout mutants: the Keio collection. Mol Syst Biol 2:2006.0008. https://doi.org/10.1038/msb4100050.

38. Castellazzi M, George J, Buttin G. 1972. Prophage induction and cell division in E. coli. I. Further characterization of the thermosensitive mutation tif-1 whose expression mimics the effect of UV irradiation. Mol Gen Genet 119:139-152. https://doi.org/10.1007/BF00269133.

39. Wang WB, Tessman ES. 1985. Evidence that the recA441 (tif-1) mutant of Escherichia coli K-12 contains a thermosensitive intragenic suppressor of RecA constitutive protease activity. J Bacteriol 163:407-409. https://doi .org/10.1128/JB.163.1.407-409.1985.

40. Johnson JE, Lackner LL, de Boer PA. 2002. Targeting of ${ }^{\mathrm{D} M i n C / M i n D}$ and ${ }^{D}$ MinC/DicB complexes to septal rings in Escherichia coli suggests a multistep mechanism for MinC-mediated destruction of nascent FtsZ rings. J Bacteriol 184:2951-2962. https://doi.org/10.1128/jb.184.11.2951-2962 2002 .

41. de Boer PA, Crossley RE, Rothfield LI. 1990. Central role for the Escherichia coli minC gene product in two different cell division-inhibition systems. Proc Natl Acad Sci U S A 87:1129-1133. https://doi.org/10.1073/pnas.87.3 .1129 .

42. Hernandez-Rocamora VM, Alfonso C, Margolin W, Zorrilla S, Rivas G. 2015. Evidence that bacteriophage lambda Kil peptide inhibits bacterial cell division by disrupting FtsZ protofilaments and sequestering protein subunits. J Biol Chem 290:20325-20335. https://doi.org/10.1074/jbc.M115 .653329 .

43. Khil PP, Camerini-Otero RD. 2002. Over 1000 genes are involved in the DNA damage response of Escherichia coli. Mol Microbiol 44:89-105. https://doi.org/10.1046/j.1365-2958.2002.02878.x.

44. Barshishat S, Elgrably-Weiss M, Edelstein J, Georg J, Govindarajan S, Haviv M, Wright PR, Hess WR, Altuvia S. 2018. OxyS small RNA induces cell cycle arrest to allow DNA damage repair. EMBO J 37:413-426. https://doi.org/ 10.15252/embj.201797651.

45. Wang X, Kim Y, Ma Q, Hong SH, Pokusaeva K, Sturino JM, Wood TK. 2010. Cryptic prophages help bacteria cope with adverse environments. Nat Commun 1:147. https://doi.org/10.1038/ncomms1146.

46. Harrison E, Brockhurst MA. 2017. Ecological and evolutionary benefits of temperate phage: what does or doesn't kill you makes you stronger. BioEssays 39:1700112. https://doi.org/10.1002/bies.201700112.

47. Dwyer DJ, Belenky PA, Yang JH, MacDonald IC, Martell JD, Takahashi N, Chan CT, Lobritz MA, Braff D, Schwarz EG, Ye JD, Pati M, Vercruysse M, Ralifo PS, Allison KR, Khalil AS, Ting AY, Walker GC, Collins JJ. 2014. Antibiotics induce redox-related physiological alterations as part of their lethality. Proc Natl Acad Sci U S A 111:E2100-E2109. https://doi.org/10.1073/ pnas.1401876111.

48. Faith JJ, Hayete B, Thaden JT, Mogno I, Wierzbowski J, Cottarel G, Kasif S, Collins JJ, Gardner TS. 2007. Large-scale mapping and validation of Escherichia coli transcriptional regulation from a compendium of expression profiles. PLoS Biol 5:e8. https://doi.org/10.1371/journal.pbio.0050008.

49. Kohanski MA, Dwyer DJ, Hayete B, Lawrence CA, Collins JJ. 2007. A common mechanism of cellular death induced by bactericidal antibiotics. Cell 130:797-810. https://doi.org/10.1016/j.cell.2007.06.049.

50. Datsenko KA, Wanner BL. 2000. One-step inactivation of chromosomal genes in Escherichia coli K-12 using PCR products. Proc Natl Acad Sci U S A 97:6640-6645. https://doi.org/10.1073/pnas.120163297.

51. Gibson DG, Young L, Chuang R-Y, Venter JC, Hutchison CA, Smith HO. 2009. Enzymatic assembly of DNA molecules up to several hundred kilobases. Nat Methods 6:343-345. https://doi.org/10.1038/nmeth.1318.

52. Addinall SG, Bi E, Lutkenhaus J. 1996. FtsZ ring formation in fts mutants. J Bacteriol 178:3877-3884. https://doi.org/10.1128/jb.178.13.3877-3884.1996. 